\title{
Evaluation of Dynamic Transients and Their Effects on Process Variables in Cutting Operations
}

\author{
E.E. Jumbo ${ }^{1}$, P.P. Jombo ${ }^{2}$, E.M. Adigio ${ }^{3}$ \\ ${ }^{1,2,3}$ Department of Mechanical and Marine Engineering, Niger Delta University, Wilberforce Island, Bayelsa \\ State, Nigeria
}

\begin{abstract}
Vibration analyses of cutting processes have been used to redefine process parameters that are required or engaged to achieve better surface integrity. Studies resulting from these findings and subsequent redesigns have majorly considered solutions to identified vibration complexities without proper consideration of the effects of dynamic and non-harmonic transients generated by these vibrations. In this paper, criticalities of cutting process variables have been investigated with particular interest on the influence of dynamic transients resulting non-conformance variability issues, which are indirectly impacted on the machine components and directly transmitted to the cutting tools, with a final destination on the workpiece. The effect of this impact on the workpiece is of critical interest to the investor, whose returns on investment is significantly dependant on the technical viability of his product for which surface finish and integrity is a cardinal requirement. Thus, the study unveils the nexus between cutting process variables and non-harmonic dynamic transients by utilizing contourized pathways as measurable mechanical energy exit points. Invariably, line traces resulting from these points were shown to connote vibration signatures on the workpiece; which implies that, as the cutting machine tends towards inoperability status, by virtue of age and frequency of deployment, the rising profile of the emergent tool signatures on the workpiece indicate the necessity for redefinitions and redesigns that must eliminate the noted non-harmonic dynamic transients.
\end{abstract}

Key words: vibration signatures, inoperability status, dynamic transients, process variables, frictional forces,

\section{Introduction}

In cutting operations, studies have shown that the geometric dimensions of machined surface are often confronted with surface integrity complications and related issues. When this happens, dimensional accuracy becomes a critical implication of non-adherence, and by extended inference, the reason for economic losses and poor technical viability. What role do dynamic transients play in this drawback and how does it affect the structural relativity and relevance of individual components and the logic of operation in association with other components. Thus, dynamic transients have to do with the following operational states and their boundary conditions: They are;

(a) stable and pseudo-stable state of operation

(b) metastable or transient excited state off operation, and

(c) Unstable state of operation.

An understanding of these operational states of cutting machines listed above makes it imperative to evaluate the various contributions of the identified transients to the perceived and observed problems encountered in surface finishing operations. Secondly, the direct effects of these operational and quasioperational states bring to fore the need to eliminate or if impossible, control or reduce vibrations and their consequences on the tool/workpiece. This is because high resonating vibration conditions can result faster tool wearing and cleavages as observed by Pavel et al [1]. Furthermore, since cutting operations are mainly hard tuning activities, it is important to state that due to operational speed requirements some activities can result into self-excited vibrations and resultant fluctuating increase in cutting energy. It is clear that vibrations are natural consequences of frictional forces undergoing static, quasi-static and dynamic transitions.

Thus, for any mass $\boldsymbol{m}$, partial or normal restriction or resistance to a new state transition causes vibration, and vibration itself generates dynamic transients which are energy transitions in form of shock waves. Studies [2] have shown that attempts to reduce these instabilities have resulted reduction in cutting efficiencies and productivity. Even though these reductions have targeted the following:

a. cutting width and chip thickness,

b. workpiece fed rate and cutting tool thrust force (tool entry force),

c. tool geometry re-characterization; 
Problems associated with the dynamic transients of the cutting machines have not been solved, as is demonstrated in this paper.

Further, the effects are only transferable to the finished products by multiplier impacts on the 'process variables' of the cutting operations. Thus, this paper has shown an in-depth study of the nature and consequences of these process variables and how they could be better optimized by the control of the identified operational states of the cutting process. The study also demonstrated the immediate and remote causes of these dynamic transients and how their 'non-conformal variabilities' constitute or result to a process argument requiring attention and resolution. In this vein, process variabilities were viewed as imposed or self-generated limitations to the quality regime. This implies that zero defect tolerance programs of manufacturing endeavours are near-impossibilities due largely to dynamic transients resulting various forms of fluctuations in cutting process conditionality.

The foregoing notwithstanding, this paper opines that an upper limit measure of importance should be attached to the dynamic characteristics of the cutting processes; most especially the range of conditions and parameters under which process consequential transients will occur, and the pattern of their occurrences. When these dynamic transients (together with their contributory factors such as, how, where, when, why etc) are understood, then it would be possible to optimize the efficiency relativities of the machinery process of cutting. However, in an attempt to solve this "dynamic error" problem, many scholars have proffered solutions which invariably terminated at the turn-milling alternative process, which has the characteristic of higher productivity. Even in this case, Pogacknik and Kopac [3] observed that the highest level of efficiency associated with turnmilling process has not been able to contend with the negative effects of the process variables problem. They referred to this problem as "process-kinematics-variations" and limited it to the entry-exit condition of the toolworkpiece interface. As would be seen later in this paper the entry-exit assumption is limited in context in the sense that dynamic patterns of behaviors of cutting machines (as in other machines) are also dynamic in nature; this is because as machines get older the performance rating are noticed to decline. In furtherance of this argument, Schulz et al [4] agreed in principle with Pogacknik and Kopac [3], but concluded that "all the disturbances and instabilities are caused by deflections in machinery systems". They however failed to show the sources of these deflections and disturbances.

The central issue in this paper is to show a link between dynamic transients and process variables during cutting operations and how these non-harmonic transients' affects or influence process variables.

\section{Dynamic Transients in Machine Tool Operations}

It should be noted that transients in machine tools equipment such as lathes and drilling machines are mainly due to feed drive conditionalities, which results non-logical operability issues, partial and full instabilities and other related dynamic behaviors. These behaviors are products of operational situations, which directly results from the interactions between internal and external forces, which are by nature incidental and coincidental frictional forces that are basically required to perform cutting operations. Thus, dynamic transients associated with machine tool operations are relative and generalized systemic consequences of the operability factors which are dependent on the performance and efficiency ratings of the machines.

This means that, a machine's operational efficiency is directly proportional to the work rate and "total accumulative machine work time". These factors possess incidental effects on the dynamic transients' incremental values as performance of the machine tends towards partial and then complete inoperability regimes. It further implies that in most machine tool operations, the extent of allowable dynamic transients determines the level of machine efficiency. With respect to this postulation, Lee and Dornfeld [5] proposed the use of various types of process-slaved sensors to measure inter-components' performance indices and characteristics. The difficulty or challenge of this measurement is the understanding of this paper that; dynamic transients of machine tools requires aggregative multi-stage analysis and evaluation which would take into cognizance the sum total of all the vibration variables incidental to that cutting operation. In furtherance of this reasoning, Hunter et al [6] conducted component by component vibration analysis for lathe TNP160A machine and generated the table below:

Table 1: Natural frequencies of parts of the TNP 160A

\begin{tabular}{|l|c|c|}
\hline \multicolumn{1}{|c|}{ Object } & Frequency $\quad H z$ & Damping \\
\hline Chuck & 315 & 0.065 \\
\hline Tail stock & 277 & 0.064 \\
\hline Saddle & 226 & 0.132 \\
\hline Head stock & 326 & 0.0708 \\
\hline
\end{tabular}

In a confirmation experiment with D360 x 1000 lathe machine, this study found that similar values applied but with a distinct deviation for headstock frequency value which recorded a high value of $364 \mathrm{~Hz}$ and 0.707 
damping factor. Thus, irrespective of the fact that the confirmation test indicated high value for the headstock, this condition is understandable. It should be noted that the headstock of lathe machines contains operational components such as gears, electric motors, drives, and cams. Furthermore, as reported by Kalpakjian and Schmid [7], 'headstocks are equipped with motors, pulleys, V-belts that supply power to the spindle at various rotational speeds. This implies that, if the speed of individual rotational member undergoing angular acceleration $\boldsymbol{\alpha}$ is summed up for all rotating members, then the assumed operational mean $\boldsymbol{\mu}_{\boldsymbol{o}}$ which generated the accumulated frequency for the headstock becomes $\sum \alpha / N$ where $N$ is indicative of the total number of the members in the headstock. It should be pointed that $\boldsymbol{\mu}_{\boldsymbol{o}}$ is only an assumed mean that is representative of varying angular velocities $\omega$ and their corresponding frequency values. Thus, this paper notes that a cumulative tendency for $\boldsymbol{\mu}_{\boldsymbol{o p}}$ results to a complicated vibration initiator referred in this paper as non-harmonic relativity and expressed as:

$\sum \alpha / N=\sum \frac{\left(\omega_{1}+\omega_{2}+\omega_{3}+\ldots \ldots+K\right.}{N}$

Where, $\mathrm{K}$ is all the analyzable components of $\omega \in \alpha$.

Thus, over an extended machine operational time in the range of $\boldsymbol{t}_{\boldsymbol{o}}, \boldsymbol{t}_{\boldsymbol{l}}$ and $\boldsymbol{t}_{\boldsymbol{1}+\boldsymbol{q}}$ the identified vibration initiator, $\sum \alpha / N$ constitutes a backlash avalanche of retributive forces that traces its weak point to the work tool where as a transient non-harmonic sink, mechanical energy exits the system. This identified exit energy as observed by Kopac and Sali [8] constitute a "specific place of frequency resonance" which indicates the actual location of post machining irregularity.

The foregoing implies that as a cutting machine gradually progresses towards inoperability due to these transients and other wear-out conditions, the transient element resulting from this condition is a diminutive vibration initiator which could be anything from illogical operational sequence to components misalignments, leveling and surface-inclination problems, tribological and other external or internal process constraints such as fed rate hysteresis-tool entry and new depth loading factors as reported in this paper.

In support of the foregoing argument, Khanfir et al [9] reported that at the point of loading a workpiece, a peak vibration effect is noticeable and that this constitutes a high frequency value which has dominant effect throughout the machining period. This paper cannot agree less, especially where Li [10] observed that varying depth of cut results varying lead angles. As shown in Fig. 1, if the depth of cut increases, it implies that tool nose radius or tool area of contact has also enlarged. The result of this development is that the periodogram for initial tool entry is approximately equal to the periodogram for a new depth of cut during the run-off of an operational period. This is because the value of the lead angles in the two scenarios is approximately the same. This means that process variables associated with cutting operations are affected by initial tool entry and increased depth of cut conditions.

Consequently, it could be further submitted that these two conditions are vibration initiators since as discussed earlier they possess the propensity for non-harmonic transient retribution in the axis and direction of the incident force $\boldsymbol{F}_{\boldsymbol{i}}$. In this regard, depth of cut has a relative effect on lead angle and an energy backlash effect at the tool point.

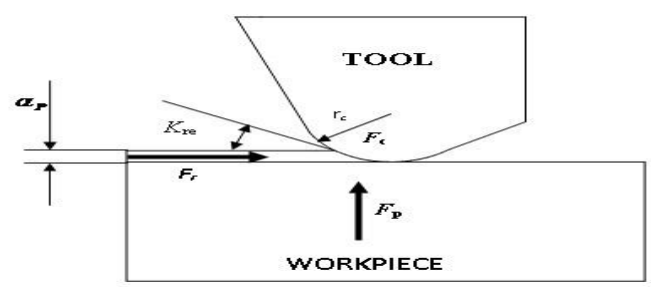

Figure 1 Schematic illustration of tool-workpiece interface geometry

\section{Dynamic Transients in Machine Tool-Workpiece Interface}

It is important to note that dynamic transients resulting from the momentary re- characterization of process variables in cutting operations can also be linked to the machine tool-workpiece interface conditionality. Interestingly, process variable re-characterization is effected by the emergent post contact conditions of the interface. This phenomenon can be better understood if consideration is made for three-dimensional cutting operations. The implication of the contact angle under three-dimensional cutting operations is that the clip flow would have a lateral direction; that is the chip would flow sideways instead of perpendicular to the tool as in orthogonal cutting. Thus, in 3-D or oblique cutting, the chip flow angle $\alpha_{c}$, the normal rake angle $\alpha_{n}$ are conditional process variables that are dependent on tool impact geometry with respect to the workpiece feed velocity $v$ and the resultant chip exit velocity $v_{c}$, thus, process oriented geometry is generated at this machine tool-workpiece interface and is characterized by an effective rake angle $\alpha_{e}$ which is subtended at the contact 
point of these two velocities, $\boldsymbol{v}$ and $\boldsymbol{v}_{\boldsymbol{c}}$. Kalpakjian and Schmid [7] also reported that since experimentally $\alpha_{\boldsymbol{c}}$ is equal to $\boldsymbol{i}$, the inclination or contact angle, the effective rake angle $\alpha_{e}$ could be determined from process conditions defined by (2),

$\boldsymbol{\alpha}_{\mathrm{e}}=\sin ^{-1}\left(\sin ^{2} \boldsymbol{i} \cos ^{2} \boldsymbol{i} \sin \boldsymbol{\alpha}_{n}\right)$

In order to sustain the argument above it should be noted that since the process variables $i$ and $\alpha_{n}$ can be measured directly, then $\alpha_{e}$ can be calculated by substitution of $i$ and $\alpha_{n}$ values into (2). By extension or extrapolation of resultant propensities, it should be stated that a functional increase in $i$ causes a derivative expansion of $\alpha_{e}$ such that:

$$
\begin{aligned}
& i \propto \alpha_{e} \text { and } \\
& i=k \alpha_{e}
\end{aligned}
$$

Where, $\boldsymbol{k}$ is a representative derivative ratio (RDR) of the machine tool contact angle and its resultant effectiveness on the workpiece when it is varied.

\section{Matrix Oriented Roughing}

Having established the foregoing, what relativity exist between the geo- structural integrity of the referenced interface with respect to the dynamic transients that have been pointed to connote ambivalent instabilities which directly impact on the prolonged efficiency and effectiveness of the machine? In response to this complex poser, recourse would be made to 3-D contour matrix oriented roughing for machined surface precision and integrity assessment.

In this assessment using $\mathrm{CNC}$ planes determination programmes, 3-D contour matrix and column vector of the machined surface could be defined on a rectangle grid coordinates which would produce contour maps with $n^{\text {th }}$ contour delineation. This delineation can also be used as tool path axis for analysis of surface finish operations.

Upon deployment of the planes determination programmes the process variable involved in cutting operations (e.g. milling) are encoded (by G-codes for CAD/CAM utility frameworks) as a matrixial function with contourized features. Thus, the contouring function resolves the input matrix as a regularly spaced grid with each element connected to its nearest neighbors and in order to calculate the contour matrix the CNC algorithm scans the input matrix and compares the values of each block of four neighboring elements (i.e. cell) in the matrix with the contour level values. If the contour level falls within the cell, the algorithm performs a liner forward and backward interpolation to locate the point at which the contour intersects and crosses the edge of the cell. Further, in order to determine the heights of the contour lines, the algorithm connects these points to produce a segment of contour lines. Thus, the algorithm produces two row of matrixes from these lines and each contour line defined in this matrix begins with a column that contains the value of the contour along the grid coordinates together with the $(x, y)$ vertices in the contour line. Fig. 2 shows the basic contouring algorithm generated from this resolution.

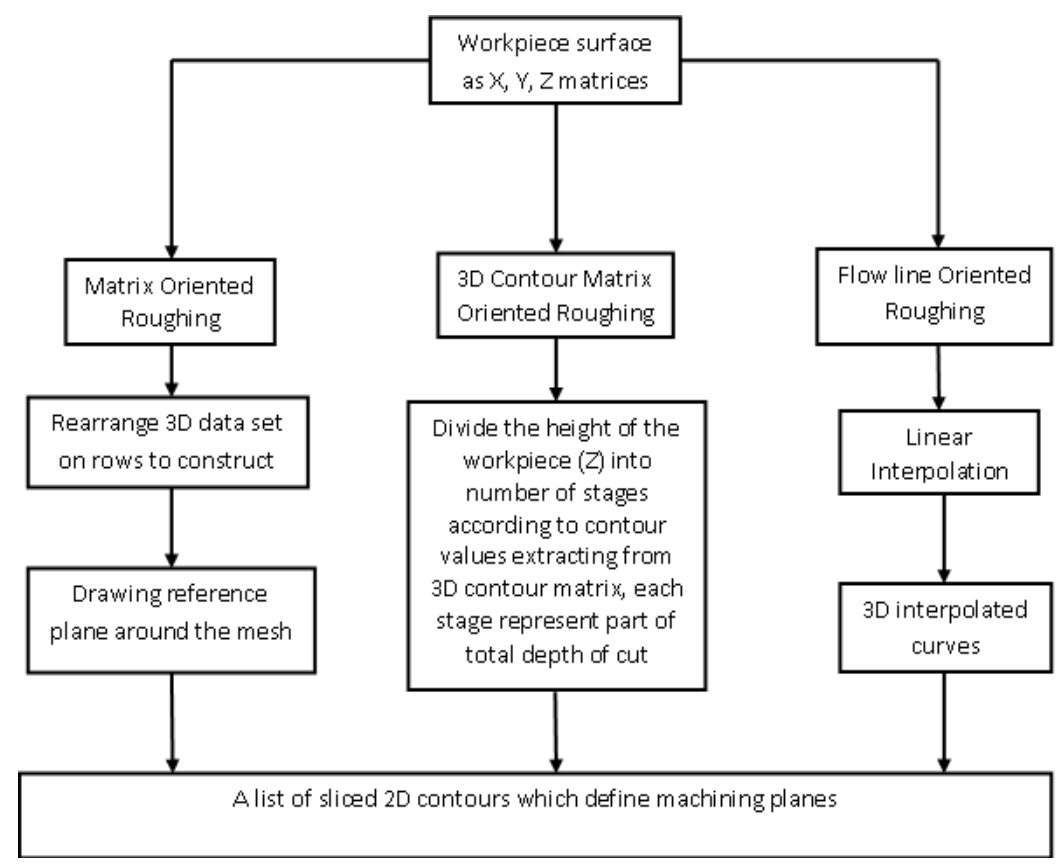

Figure 2 Contouring algorithm for cutting operations 
In view of this development, Mulcaly [11] reported that this method of surface determination can also be achieved by a mathematical sequence for which a plot on rectangular grid coordinates in the U-W plane utilizing values in a 3-D space could be generated from the relation;

$$
S\{U, \omega\}=\sum_{i, j=0}^{3}\left(i^{3}\right)\left(j^{3}\right) U^{i}(1-U)^{3-i} \omega^{i}(1-\omega)^{3-i} P^{i j}
$$

Where, $(0 \leq i, j \geq 3), U, \omega \in[0,1], P_{j}$ are points of the surface $S(U, \omega)$.

\section{Flow Line Oriented Rouging}

The foregoing matrix oriented roughing is deterministic for coordinate point assessment; however it is of necessity that the lines where these points are located are determined. This is achieved by flow line oriented roughing which is used to generate tool path for CNC 3-D roughing process. This sequence involves the determination of cutter paths by indexing along the U-W parameters of parametrically defined surface. When this is defined, the tool follows the natural parametric flow lines of the surface. Hughes et al [12] pointed that flow line oriented roughing can be mathematically customized by utilizing the Cubic Bezier curve. The process parameter for this curve with respect to each flow line becomes;

$$
P(u)=\sum P_{i} P_{i, n}(u)
$$

Where, $P_{i, n}(u)=\frac{n ! u^{i}(1-u)^{n-1}}{i !(n-i) !}, \mathrm{u} \in[0,1], \mathrm{N}$ is number of control points and $\mathrm{P}_{\mathrm{i}}$ are end points for curve segment

However, it should be noted that there is a process caveat which limits this model to single path surfaces.

\section{Discussions}

It has been established that dynamic transients are products of vibrations which results from frictional forces generated and required for machines to work. When these vibration transients instantaneously accumulate, an energy sink in the form of an exit point is required in order to vent the energy. Invariably, the tool point becomes a significantly noted non-harmonic transient discharge centre. The foregoing notwithstanding, the effect of these dynamic transients can be evaluated both empirically and theoretically. In order to achieve this, the paper utilized the resources of MATHLAB R.12, V.6.0 to apply the discussed 3-D Contour Matrix Oriented Roughing. Thus, graphical display for these applications are set in 3-D coordinate forms in a $x y z$ space plane and used to generated G-code programs for surface profile evaluation.

It should be noted that the cutting process implication and relevance of these graphs indicate that the exit of dynamic transients from a cutting process using machine tool-workpiece interface as a discharge point, generates aggregated contour forms which is specific for 3-D contour matrix oriented operations (as a method of evaluation) and specific for flowline machining path.

\section{Conclusion}

This paper has observed that since the machine tool must make contact with the workpiece, then the exiting dynamic transients of the machine would also impact on the work piece during the cutting operation of the tool.

Thus, in both cases discussed, the axis of the $x, y, z$ plane are the same and the resultant graph in 3-D space could be reduced to a 2-D crest and trough energy discharge profile of a sinusoidal waveform; indicating a cycle of mechanical energy exit or discharge in the form of dynamic transients which are impacted on the workpiece (as have been pointed) and could be traced as the tool path on the workpiece. Investigation however shows that this tool path trace could be seen on the post machined workpiece profile as contour lines representing mechanical energy or vibration transients discharge points.

In addition, since the surface integrity of the work piece is the central issue, the process variables indicated in this paper are influenced by these transients. Thus, as the cutting machine ages, the effects of these dynamic transients become more significant. The implication on surface integrity of a machined profile is that of non-conformance to specification and poor surface integrity.

\section{Acknowledgement}

The Niger Delta University Bayelsa State, Nigeria is acknowledged for financial support. We particularly thank Prof. O.O. Onyemaobi for patience and unfailing encouragement. 


\section{References:}

[1] Pavel R., Marinescu I., Deis M., Pillar J., Effect of tool wear on surface finish for a case of continous and interrupted hard turning, Journal of Materials processing Technology, 170 (2005) 341-349.

[2] Chein-Shan L., Coulomb Friction Applied to Analyze the Cutting Vibration of Tool on Workpiece, Journal of Marine Science and Technology, Vol. 9, No. 2, (2001), 122-129

[3] Pogacnik M., and Kopac J., Dynamic Stabilization of the Turn Milling Process by Parameter Optimization, Proc Instn of Mec Engrs, Vol 214 part b, ImechE (2000), 127-135

[4] Schulz H., Stoic A. and Sahm A., Improvement of Cutting Process in Accordance with Process Disturbances, $7^{\text {th }}$ International Conference on Production Engineering CIM2001, HUPS Zagreb (2001), 1123-1131

[5] Lee Y., and Dornfeld D.A., Application of Open Achitecture Precision System Machining. $31^{\text {st }}$ CIRP International Seminar on Manufacturing Systems, Berkeley, CA, May (1998), 463-441.

[6] Hunter R., Vizan A., Perez J., and Rios J., Knowledge Model as an Integral Way to reuse the Knowledge for fixture of design process, International Confernce on advances in Materials and Process Technology, AMPT' @005, Gliwice-Wisla, (2003), 254-257

[7] Kalpakjian S., and Schmid S.R., Manufacturing Engineering and Technology, $4^{\text {th }}$ Edition, Pearson Education, NJ, (2001), 563-564

[8] Kopac J. and Sali S., Tool Wear Monitoring during the Turning Process, Journal of materials processing Technology 113 920010, (2001), 312-316

[9] Khanfir H., Bonis M. and Revel P., Improving Waviness in Ultra Precision Turning by Optimizing the Dynamic Behavior of a Spindle with Magnetic Bearings, International Journal machine Tools and Manufacture 45 (2005), 841-848

[10] Li X. Real-Time Prediction of Workpiece Errors for a CNC Turning Centre, part 3. Cutting Force Estimation using sensors, International Journal of Advanced Manufacturing Technology17 92001, (2001), 659-664

[11] Mulcahy C., The Basic Curves and Surfaces of Computer Aided Geometric Design, Department of Mathematics, Spelman College, Atlanta, USA, 4. (2011)

[12] Hughes J. and Philips R.L Introduction to Computer Graphics, Addison-Wesley Publishing Company, Ontario, 1994. 